\title{
Detection of giant pulses in pulsar PSR J1752+2359
}

\author{
A. A. Ershov ${ }^{1,2}$ and A. D. Kuzmin ${ }^{1,2}$ \\ 1 Pushchino Radio Astronomy Observatory, Astro Space Center, Lebedev Physical Institute, Russian Academy of Sciences, \\ Pushchino 142290, Russia \\ e-mail: ershov@prao.psn.ru \\ 2 Isaac Newton Institute, Chile, Pushchino Branch, Russia
}

Received 29 November 2004 / Accepted 20 July 2005

\section{ABSTRACT}

We report the detection of Giant Pulses (GPs) in the pulsar PSR J1752+2359. About one pulse in 270 has a peak flux density more than 40 times the peak flux density of an average pulse (AP), and the strongest GP is as large as 260. The energy of the strongest GP exceeds the energy of the average pulse by a factor of 200 which is greater than in other known pulsars with GPs. PSR J1752+2359 as well as the previously detected pulsars PSR B0031-07 and PSR B1112+50, belong to the first group of pulsars found to have GPs without a strong magnetic field at the light cylinder.

Key words. stars: neutron - stars: pulsars: general - stars: pulsars: individual: PSR J1752+2359

\section{Introduction}

Giant pulses (GPs) are short duration burst-like increases of the intensity of individual pulses from pulsars.

The peak intensities and energies of GPs greatly exceed the peak intensity and energy of the average pulse (AP). The energy distribution of GPs follows a power-law. The GPs are much narrower than the APs and their phases stably placed within the APs.

This rare phenomenon was first detected in the Crab pulsar PSR B0531+21 (Staelin \& Sutton 1970) and the millisecond pulsar PSR B1937+21 (Wolszczan et al. 1984), both with very strong magnetic fields on the light cylinder of $B_{\mathrm{LC}}=$ $10^{4}-10^{5} \mathrm{G}$. This gave rise to the suggestion that GPs occur in pulsars with very strong magnetic fields at the light cylinder and a search of GPs was oriented towards those pulsars. As a result GPs were detected in five other pulsars with very strong magnetic fields at the light cylinder: PSR B0218+42 (Joshi et al. 2003), PSR B0540-69 (Johnston \& Romani 2003), PSR B1821-24 (Romani \& Johnston 2001), PSR J1823-3021 (Knight et al. 2005) and PSR B1957+20 (Joshi et al. 2003).

We report the detection of GPs in the pulsar PSR J1752+2359. Correlating this with our previously published data on PSR B1112+50 (Ershov \& Kuzmin 2003) and PSR B0031-07 (Kuzmin et al. 2004), we show that GPs exist in pulsars with relatively low magnetic fields at the light cylinder of $B_{\mathrm{LC}}=1-100 \mathrm{G}$.

The detection of GPs in PSR J1752+2359 is a result of our monitoring program for GPs. More details will be published upon completion of this program.

\section{Observations}

Observations were performed from December 24, 2003 to October 04, 2004 with the Large Phase Array (BSA) Radio Telescope at Pushchino Radio Astronomy Observatory of the Lebedev Physical Institute at a frequency of $111 \mathrm{MHz}$. BSA is a transit telescope with an effective area of about 15000 square meters. One linear polarization was received. We used a 128 -channel receiver with a channel bandwidth of $20 \mathrm{kHz}$. The sampling interval was $2.56 \mathrm{~ms}$ and the receiver time constant was $\tau=3 \mathrm{~ms}$.

All observations were time-referenced to the Observatory's rubidium master clock, which in turn was monitored against the National Time standard via TV timing signals. During the offline data reduction, the signal records were cleaned of radio interferences. Subsequently, the inter-channel dispersion delays imposed by the interstellar medium were removed. Each observation was analyzed for pulses with amplitudes exceeding a preset level, and its amplitude, pulse width and phase were derived.

For verification that GPs were actually detected we used similar detection technique to McLaughlin \& Cordes (2003) and Cordes et al. (2004) for the detection of GPs. Our processing consisted of: 1) checks for radio interference; 2) dedispersing by summing over frequency channels while taking into account the time delay associated with plasma dispersion in the interstellar matter; 3) averaging the time series synchronously with the pulsar period to form a standard intensity profile; 4) identifying individual giant pulses and their occurrence times by selecting intensity samples that exceeded the 


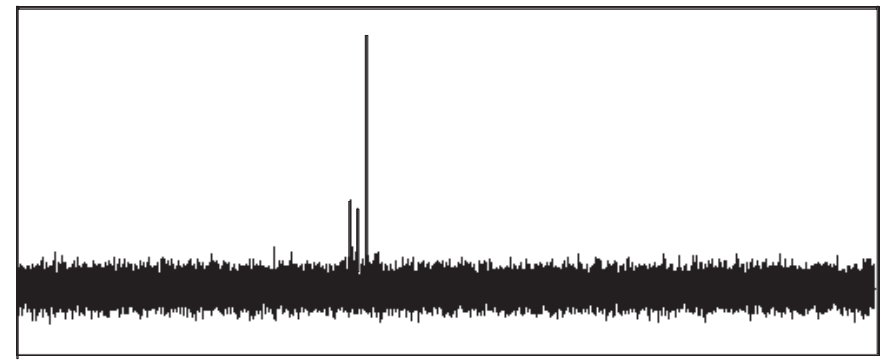

Fig. 1. One observation session of GPs. Three large pulses stand out of the noise background and weak pulses are observed inside 420 pulsar periods.

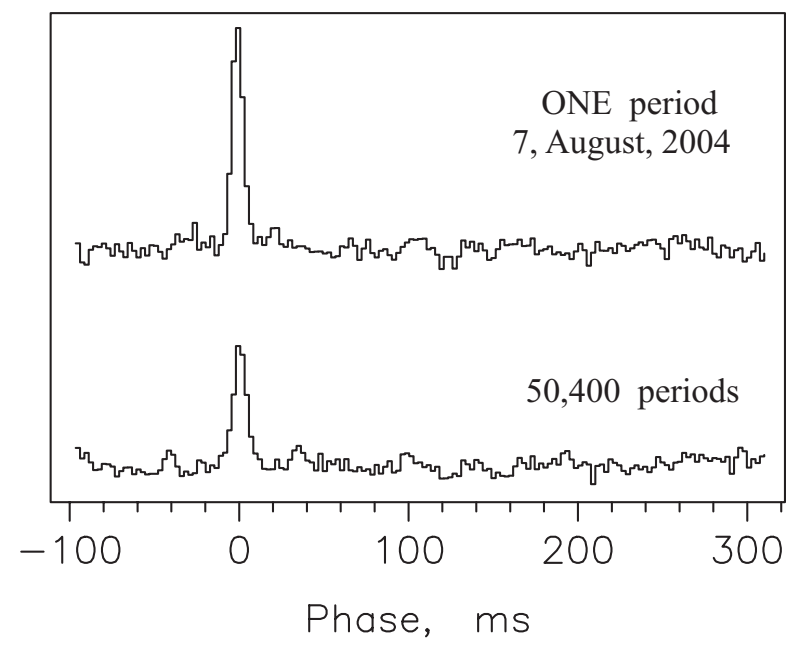

Fig. 2. (Top) The strongest observed pulse. (Bottom) The average pulse profile containing 50400 pulsar periods. The intensity of the profiles is shown in arbitrary units.

off-pulse mean by 5 sigma; 5) aligning average profiles and individual giant pulse profiles in pulse phase with the TimApr ${ }^{1}$ program. For the TimApr analysis, we used the timing model from Lewandowski et al. (2004).

\section{Results}

Only the brightest pulses are well separated from the underlying pulse and noise fluctuations. They are both single pulses and groups of several strong pulses. Figure 1 shows an example of one observation session of GPs. A group of three bright pulses standing out of the noise background and underlying weak pulses were observed inside 420 pulsar periods. The 187 pulses (1 pulse for 270 observed periods) with $S / N \geq 5$ were selected and analyzed. The observed peak flux density exceeded the peak flux density of the average pulse (AP) (which is equivalent to the integrated profile) by more than a factor of 40. Figure 2 shows the strongest observed GP together with the AP averaged over 50400 pulsar periods.

The value of the GP flux density was determined relative to the AP flux density as

$S_{\text {peak }}^{\mathrm{GP}}=S_{\text {peak }}^{\mathrm{AP}} \times\left(I^{\mathrm{GP}} / I^{\mathrm{AP}}\right)$,

\footnotetext{
${ }^{1}$ psun32.prao.psn.ru/olegd/soft.html
}

where $I^{\mathrm{GP}} / I^{\mathrm{AP}}$ is the ratio of the GP to AP peak intensity and $S_{\text {peak }}^{\mathrm{AP}}$ is the peak flux density of AP

$S_{\text {peak }}^{\mathrm{AP}}=S_{\text {mean }}^{\mathrm{AP}} / k_{\text {form }}$,

where $S_{\text {mean }}^{\mathrm{AP}}$ is the flux density averaged over a pulsar period $P$, $k_{\text {form }}=w_{\text {eff }} / P$ is the duty cycle of the pulsar, and $w_{\text {eff }}-$ the effective pulse width.

The value of $S_{\text {mean }}^{\mathrm{AP}}$ was measured relative to the reference discrete source $3 \mathrm{C} 452$, and to the reference pulsars PSR B0329+54, B0809+74, B0823+26, B0834+05, B0919+06, B1541+09, B1839+56 and B1919+21 with known flux densities.

To normalize the observation system's parameters and control its stability, we have performed control observations of the reference pulsar PSR B1919+21 throughout the same time interval as the GPs. Its flux density, measured in the same way as for PSR J1752+2359, is stable within 25 percent. We calculate the AP of the total intensity by summing six partial-sum profiles of 20 observation sessions ( 8400 pulsar periods) in which signal-to-noise ratio was larger than five. These samples revealed similar values of flux density, within 15 percent. The AP mean flux density of PSR J1752+2359 was $S_{1752}=11 \pm 4 \mathrm{mJy}$.

Interstellar scintillations (ISS) do not significantly affect our intensity measurements. At the frequency of our observations $(111 \mathrm{MHz})$, the time scales of the refractive ISS for both $\mathrm{J} 1752+2359$ and PSR B1919+21 (DM = 36 and $\left.12.4 \mathrm{pc} \mathrm{cm}^{-3}\right)$ are about $1 \mathrm{~min}$ (Malofeev et al. 2004), and smoothed over the duration of our observation of several minutes. The long term refractive ISS are weak and do not seriously affect the intensity measurements.

The peak flux density of the AP is $S_{\text {peak }}=S_{1752} / k_{\text {form }}$, the pulsar period is $P=409 \mathrm{~ms}$ and the effective width of the AP is $w_{\text {eff }}=11.3 \mathrm{~ms}$. Thus $k_{\text {form }}=0.0276$ and the peak flux density of the AP is $S_{\text {peak }}=400 \mathrm{mJy}$.

The observed peak flux density of the strongest GP exceeds the peak flux density of the AP by a factor of 260 . Therefore its peak flux density is $S_{\text {peak }}^{\mathrm{GP}}=105 \mathrm{Jy}$.

The energy of the strongest observed GP is $S_{\text {peak }}^{\mathrm{GP}} \times w_{\text {eff }}=$ $920 \mathrm{Jy} \mathrm{ms}$, where $w_{\mathrm{eff}}^{\mathrm{GP}}=8.8 \mathrm{~ms}$ is the observed width of the GP. The energy of AP is $E=S_{1752} \times P=4.5 \mathrm{Jy} \mathrm{ms}$. Then, the observed energy of the GP exceeds the energy of the AP by factor of 200. This is the most pronounced energy increase factor $k_{\mathrm{E}}=E^{\mathrm{GP}} / E^{\mathrm{AP}}$ among the known pulsars with GPs. A pulse whose energy exceeds the energy of the average pulse (AP) by more than a factor of 100 is encountered approximately once in 3000 observed periods.

Lewandowski et al. (2004) show the bursting behaviour of this pulsar, which spend $70-80 \%$ of the time in a "quasi-null" state. The amplitude of the AP formed only of "burster" pulses will be about 4 times stronger that the AP obtained by folding of all observed pulses. In this case the ratio of the peak flux densities $S_{\text {peak }}^{\mathrm{GP}} / S_{\text {peak }}^{\mathrm{AP}}$ and energies $E^{\mathrm{GP}} / E^{\mathrm{AP}}$ of the GP to the AP will be about 4 times less. Even so, the observed energy of the GP exceeds the energy of the AP by factor of 50, that is nearly the same as for classical GPs in the Crab pulsar (see Table 1). 
Table 1. The energy properties of giant pulses.

\begin{tabular}{lcrccccccl}
\hline \hline PSR & $\begin{array}{c}B_{\mathrm{LC}} \\
{[\mathrm{G}]}\end{array}$ & $\begin{array}{r}\text { Period } \\
{[\mathrm{sec}]}\end{array}$ & $\begin{array}{c}\text { Freq } \\
{[\mathrm{MHz}]}\end{array}$ & $\begin{array}{c}E^{\mathrm{AP}} \\
{[\mathrm{Jy} \mathrm{ms}]}\end{array}$ & $\begin{array}{c}S_{\text {peak }}^{\mathrm{GP}} \\
{[\mathrm{Jy}]}\end{array}$ & $\begin{array}{c}W^{\mathrm{GP}} \\
{[\mathrm{ms}]}\end{array}$ & $\begin{array}{c}E^{\mathrm{GP}} \\
{[\mathrm{Jy} \mathrm{ms}]}\end{array}$ & $E^{\mathrm{GP}} / E^{\mathrm{AP}}$ & References \\
\hline $\mathrm{J} 1752+2359$ & 71 & 0.409 & 111 & 4.5 & 105 & 8.8 & 920 & 200 & this paper \\
\hline B0031-07 & 7.0 & 0.943 & 111 & 330 & 530 & 5 & $2.5 \times 10^{3}$ & 8 & Kuzmin et al. (2004) \\
& & & 40 & 460 & $1.1 \times 10^{3}$ & 6 & $6.6 \times 10^{3}$ & 14 & Kuzmin \& Ershov (2004) \\
\hline $\mathrm{J} 0218+4232$ & $3.2 \times 10^{5}$ & $2.32 \times 10^{-3}$ & 610 & - & - & - & 1.34 & 51 & Joshi et al. (2003) \\
\hline B0531+21 & $9.8 \times 10^{5}$ & $3.31 \times 10^{-2}$ & 594 & 6.4 & $150 \times 10^{3}$ & $5 \times 10^{-4}$ & 75 & 12 & Kostyuk et al. (2003) \\
& & & 2228 & 0.11 & $18 \times 10^{3}$ & $5 \times 10^{-4}$ & 9 & 80 & Kostyuk et al. (2003) \\
\hline B1112+50 & 4.2 & 1.656 & 111 & 90 & 180 & 5 & 1440 & 16 & Ershov \& Kuzmin (2003) \\
\hline B1821-24 & $7.4 \times 10^{5}$ & $3.05 \times 10^{-3}$ & 1517 & $9.3 \times 10^{-3}$ & - & - & 0.76 & 81 & Romani \& Johnston (2001) \\
\hline J1823-3021 & $2.5 \times 10^{5}$ & $5.44 \times 10^{-3}$ & 685 & - & 45 & 0.020 & - & 64 & Knight et al. (2005) \\
& & & 1400 & - & 20 & 0.007 & - & 28 & Knight et al. (2005) \\
\hline B1937+21 & $1.0 \times 10^{6}$ & $1.56 \times 10^{-3}$ & 111 & 6.0 & - & - & 350 & 60 & Kuzmin \& Losovsky (2002) \\
& & & 430 & 0.4 & - & - & 7.5 & 19 & Kinkhabwala \& Thorset (2000) \\
& & & 1400 & 0.025 & - & - & 1 & 40 & Kinkhabwala \& Thorset (2000) \\
\hline B1957+20 & $3.8 \times 10^{5}$ & $1.61 \times 10^{-3}$ & 610 & - & - & - & 0.925 & 129 & Joshi et al. (2003)
\end{tabular}

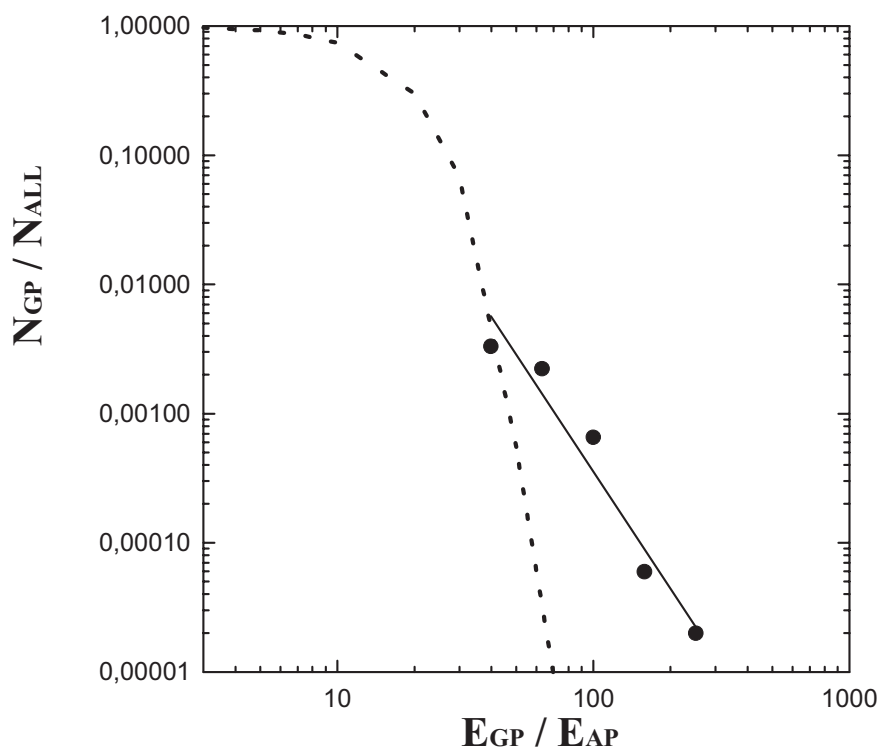

Fig. 3. The cumulative distribution of the observed GP energy $E^{\mathrm{GP}}$ as related to the AP energy $E^{\mathrm{AP}}$. The solid line is the observed power-law distribution $N^{\mathrm{GP}} / N^{\mathrm{All}} \propto\left(E^{\mathrm{GP}} / E^{\mathrm{AP}}\right)^{\alpha}$ with index $\alpha=-3.0 \pm 0.4$. The dotted line represents the possible version of the Gaussian distribution $N / N^{\mathrm{All}}=\exp \left(-a\left(E / E^{\mathrm{AP}}\right)^{2}\right)$.

Along with a large intensity, the distinguishing characteristic of the previously known pulsars with GPs is their twomode pulse intensity distribution. At low intensities, the pulse strength distribution is Gaussian, but above a certain threshold the pulse strength distribution is roughly a power-law.

In Fig. 3 we show the measured cumulative distribution of the ratio of the observed GP energy to the AP energy $E_{\text {peak }}^{\mathrm{GP}} / E_{\text {peak }}^{\mathrm{AP}}$ of all pulses analyzed. In the interval of $E^{\mathrm{GP}} / E^{\mathrm{AP}}$ from 40 to 200 , the distribution is fit by a power-law dependence $N^{\mathrm{GP}} / N^{\mathrm{All}} \propto\left(E^{\mathrm{GP}} / E^{\mathrm{AP}}\right)^{\alpha}$ with index $\alpha=-3.0 \pm 0.4$.
For $E^{\mathrm{GP}} / E^{\mathrm{AP}}$ less than 40 the signal-to-noise ratio is less than 5 , and the observed distribution is masked by noise. For this region we plot one of the possible versions of the Gaussian distribution $N / N^{\mathrm{All}}=\exp \left(-a\left(E / E^{\mathrm{AP}}\right)^{2}\right)$, which is tangent to the observed power-law distribution.

The intrinsic fine structure of GPs is masked by dispersion pulse broadening $\Delta t_{\mathrm{DM}}=4.4 \mathrm{~ms}$ and a receiver time constant $\tau=3 \mathrm{~ms}$. Therefore, from October 5 through October 25, 2004 , we performed additional observations with higher temporal resolution. We used a 128-channel receiver with a channel bandwidth of $1.25 \mathrm{kHz}$, sampling interval 0.81 , and time constant $\tau=1 \mathrm{~ms}$. In this mode, we performed 14 observation sessions containing 6800 pulsar periods.

Figure 4a shows the high resolution GP (bold line) together with the AP (dotted line). The observed peak flux density of this GP exceeds the peak flux density of the AP by a factor of 320. The plot of the AP is presented on a 250 times larger scale, and flux densities of the observed GP and AP are shown separately, on the left and right sides of the " $y$ "-axis.

The observed width of the GPs is $w_{0.5}^{\mathrm{GP}}=1 \mathrm{~ms}$, which is narrower than the AP by a factor of about 10 .

Figure $4 \mathrm{~b}$ shows the phases of the GPs whose intensities exceeded the AP by a factor of 100 . GPs cluster in a narrow phase window near the middle of the AP. The clustering is closer for stronger GPs.

The brightness temperature of the GPs is

$T_{\mathrm{B}}=S \lambda^{2} / 2 k \Omega$,

where $\lambda$ is the radio wavelength, $k$ is the Boltzmann constant, and $\Omega$ is the solid angle of the radio emission region. Adopting $\Omega \simeq(l / d)^{2}$, where $l$ is the size of the radio emission region and $l \leq c \times w_{0.5}$, where $c$ is the speed of light and the distance to the pulsar $d=2.7 \mathrm{kpc}$ (Lewandowski et al. 2004), we obtain $T_{\mathrm{B}} \geq 2 \times 10^{28} \mathrm{~K}$. 


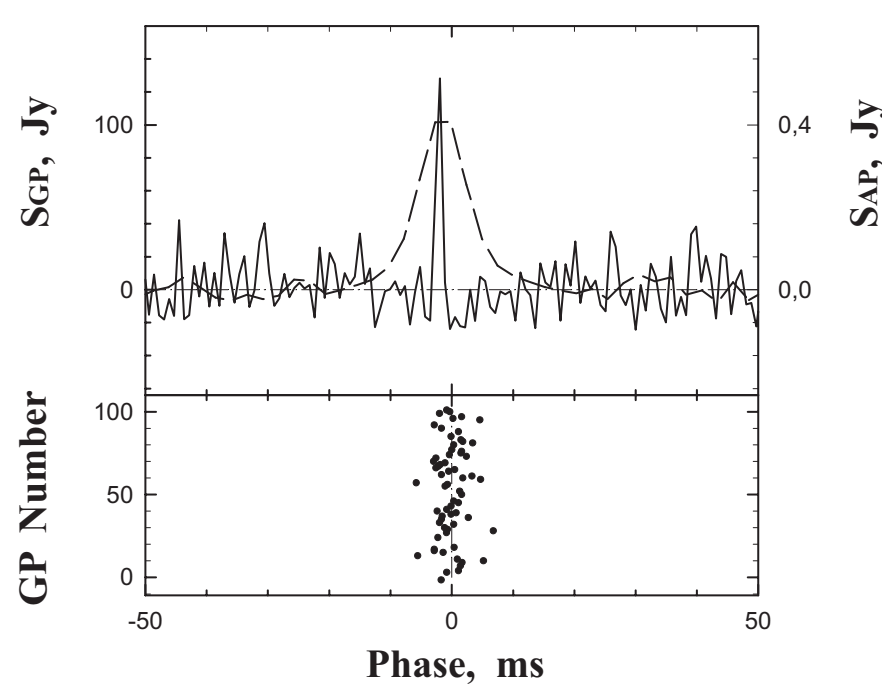

Fig. 4. (Top) The observed high resolution GP (bold line) and the AP (dotted line). The observed peak flux density of this GP exceeds the peak flux density of the AP by a factor of 320 . The plot of the AP is presented on a 250 times larger scale and flux densities of the observed GP and AP are shown separately on the left and right sides of the " $y$ "-axis. (Bottom) The phases of the GPs.

\section{Discussion}

The GPs that we detected in PSR J1752+2359 exhibit all characteristic features of the classical GPs in PSR B0531+21 and PSR B1937+21.

The peak intensities of the GPs exceed the peak intensity of the AP by more than a factor of 50. The histograms of the flux density have a power-law distribution. The GPs are much narrower than the AP and their phases are stable inside the integrated profile.

The most important aspect of this paper is the fact that PSR $\mathrm{J} 1752+2359$ (as well as PSR B0031-07 and PSR B1112+50) represent pulsars with a relatively low magnetic field $B_{\mathrm{LC}}$ at the light cylinder. This is in contrast to the canonical suggestion that GPs occur in pulsars with strong magnetic fields at the light cylinder (e.g. Romani \& Johnston 2001). The detection and first searches for GPs were performed in pulsars with extremely high magnetic fields at the light cylinder of $B_{\mathrm{LC}}=10^{4}-10^{5} \mathrm{G}$. Then it was suggested that GPs originate near the light cylinder (Istomin 2004). However, detection of GPs in the pulsars PSR B1112+50 (Ershov \& Kuzmin 2003), PSR B0031-07 (Kuzmin et al. 2004) and presently reported GPs in PSR J1752+2359 have revealed that GPs exist also in pulsars with ordinary magnetic fields at the light cylinder of $B_{\mathrm{LC}}=1-100 \mathrm{G}$. These GPs may be associated with the inner gap emission region (Gil \& Melikidze 2004; Petrova 2004).

In Table 1 we summarize the comparative data on the energy properties of giant pulses, for which the data on $E_{\mathrm{GP}}$ or $E^{\mathrm{GP}} / E^{\mathrm{AP}}$ has been published or may be derived. All known GPs, both with high and low magnetic field at the light cylinder, have the same order of magnitude energy increasing factor $k_{\mathrm{E}}=E^{\mathrm{GP}} / E^{\mathrm{AP}}$. Moreover, the presently reported GPs of PSR J1752+2359 with relatively low magnetic field at the light cylinder have the most pronounced $k_{\mathrm{E}} \cong 200$ among known pulsars with GPs.

An interesting aspect is the fine structure of GP radio emission. Giant pulses from the Crab pulsar PSR B0531+21 (Sallmen et al. 1999; Jessner et al. 2005) and LMC pulsar PSR B0540-69 (Johnston \& Romany 2003) frequently show a double structure of GPs. Kuzmin \& Ershov (2004) have detected a double structure of GPs from the pulsar PSR B0031-07 and argued that the pulses come from the polar region and not near the outer magnetosphere. In contrast Kinkhabwala \& Thorsett (2000) reported that after inspecting many pulses from PSR B1937+21 they see no evidence for multiple-peaked emission. Soglasnov et al. (2004) also claimed that GPs from this pulsar occur, in general, with a single spike.

In our observations of PSR J1752+2359 we used a 128-channel receiver with a channel bandwidth of $20 \mathrm{kHz}$. In this mode the intrinsic fine structure of GPs was masked by the dispersion broadening $\Delta t_{\mathrm{DM}}=4.4 \mathrm{~ms}$ and receiver time constant $\tau=3 \mathrm{~ms}$ which makes it hard to look for a double structure of GPs.

We have attempted to look for a fine structure of GPs in the additional observations with higher temporal resolution using 128-channel receiver with a channel bandwidth of $1.25 \mathrm{kHz}$, sampling interval $0.81 \mathrm{~ms}$ and time constant $\tau=1 \mathrm{~ms}$. But in this mode the radio telescope sensitivity was worse by factor of 4 and we performed only 14 observation sessions containing 6800 pulsar periods against 120 observation sessions containing 50400 pulsar periods in the main mode. Therefore in the high temporal resolution mode we detected only 5 GPs, but no double structure.

One should note that the GPs of PSR J1752+2359 with relatively low magnetic field at the light cylinder was observed at the low frequency of $111 \mathrm{MHz}$, whereas GPs of pulsars with strong magnetic fields at the light cylinder were observed mainly at high frequencies. It would be of interest to observe GPs of PSR J1752+2359 at high frequencies, where one can obtain better temporal resolution.

\section{Conclusions}

Giant Pulses (GPs) from pulsar PSR J1752+2359 have been detected. The energy of the GPs exceeds the energy of the average profile by a factor of up to 200, which is greater than in other known pulsars with GPs. The cumulative distribution is fit by a power-law with index $-3.0 \pm 0.4$. PSR J1752+2359 as well as the previously detected PSR B0031-07 and PSR B1112+50 are the first pulsars with GPs that do not have a high magnetic field at the light cylinder.

Acknowledgements. We wish to thank V. V. Ivanova, K. A. Lapaev \& A. S. Aleksandrov for assistance during observations. This work was supported in part by the Russian Foundation for Basic Research (project No. 05-02-16415) and the Program of the Presidium of the Russian Academy of Sciences "Non-steady-state Processes in Astronomy". We are grateful to Dr. Steve Shore for valuable comments and suggestions. 


\section{References}

Ershov, A. A., \& Kuzmin, A. D. 2003, Pis'ma v AZh, 29, 111 (Astr. Lett., 29, 91)

Cordes, J. M., Bhat, N. D. R., Hankins, T. H., McLaughlin, M. A., \& Kern, J. 2004, ApJ, 612, 375

Gil, J., \& Melikidze, G. I. 2004, in Young Neutron Stars and Their Environments, ed. F. Camilo, \& B. M. Gaensler (San Francisco: ASP), IAU Symp., 218, 321

Hankins, T. H., Kern, J. S., Weatherall, J. C., \& Eilek, J. A. 2003, Nature, 422, 141

Istomin, Y. N. 2004, in Young Neutron Stars and Their Environments, ed. F. Camilo, \& B. M. Gaensler (San Francisco: ASP), IAU Symp., 218, 369

Jessner, A., Klein, B., Hankins, T., et al. 2005, 35th COSPAR Scientific Assembly, 18-25 July 2004, Paris, France, 905

Johnston, S., \& Romani, R. W. 2003, ApJ, 590, L95

Joshi, B. C., Kramer, M., Lyne, A. G., McLaughlin, M., \& Stairs, I. H. 2004, in Young Neutron Stars and Their Environments, ed. F. Camilo, \& B. M. Gaensler (San Francisco: ASP), IAU Symp., 218,319

Knight, H. S., Bailes, M., Manchester, R. N., \& Ord, S. M. 2005 ApJ, 625,951
Kostyuk, S. V., Kondratiev, V. I., Kuzmin, A. D., Popov, M. V., \& Soglasnov, V. A. 2003, Pis'ma v AZh, 29, 440 (Astr. Lett., 29, 387)

Kinkhabwala, A., \& Thorsett S. E., 2000, ApJ, 535, 365

Kuzmin, A. D., \& Losovsky, B. Ya. 2002, Pis'ma v AZh, 28, 25 (Astr. Lett., 28, 21)

Kuzmin, A. D., Ershov, A. A., \& Losovsky, B. Ya. 2004, Pis'ma v AZh, 30, 285 (Astr. Lett., 30, 247)

Kuzmin, A. D., \& Ershov, A. A. 2004, A\&A, 427, 575

Lewandowski W., Wolszczan A., Feiler G., Konacki M., \& Soltysinski T. 2004, ApJ, 600, 905

Malofeev, V. M., Malov, O. I., \& Shchegoleva, N. V. 2000, AZh, 77, 499 (ARep, 44, 436)

McLaughlin, M. A., \& Cordes, J. M. 2003, ApJ, 596, 982

Petrova S. A. 2004, A\&A, 424, 227

Romani, R. W., \& Johnston, S. 2001, ApJ, 557, L97

Sallmen, S., Backer, D. C., Hankins, T. H., Moffett, D., \& Lundgren, S. 1999, ApJ, 517, 460

Soglasnov, V. A., Popov, M. V., Bartel, N., et al. 2004, ApJ, 616, 439

Staelin, D. H., \& Sutton, J. M. 1970, Nature, 226, 69

Wolszczan, A., Cordes, J. M., \& Stinebring, D. R. 1984, in Millisecond Pulsars, ed. S. P. Reynolds, \& D. R. Stinebring (NRAO, Green Bank), 63 